\title{
Autophagy in immunity and inflammation
}

\author{
Beth Levine ${ }^{1,2,3}$, Noboru Mizushima ${ }^{4} \&$ Herbert W. Virgin ${ }^{5}$
}

Autophagy is an essential, homeostatic process by which cells break down their own components. Perhaps the most primordial function of this lysosomal degradation pathway is adaptation to nutrient deprivation. However, in complex multicellular organisms, the core molecular machinery of autophagy - the 'autophagy proteins' - orchestrates diverse aspects of cellular and organismal responses to other dangerous stimuli such as infection. Recent developments reveal a crucial role for the autophagy pathway and proteins in immunity and inflammation. They balance the beneficial and detrimental effects of immunity and inflammation, and thereby may protect against infectious, autoimmune and inflammatory diseases.

T There is only one known mechanism that eukaryotic cells possess to dispose of intracellular organelles and protein aggregates that are too large to be degraded by the proteasome. It is therefore not surprising that this mechanism - the lysosomal degradation pathway known as autophagy - is also used to degrade microorganisms (such as viruses, bacteria and protozoa) that invade intracellularly ${ }^{1,2}$. Indeed, the mutation of autophagy genes increases susceptibility to infection by intracellular pathogens in organisms ranging from plants to flies to worms to mice, and possibly to humans. Perhaps less apparent, but teleologically as intuitive, the autophagy pathway or unique functions of autophagy proteins also have a central role in controlling other diverse aspects of immunity in multicellular organisms.

The autophagy machinery is thought to have evolved as a stress response that allows unicellular eukaryotic organisms to survive during harsh conditions, probably by regulating energy homeostasis and/ or by protein and organelle quality control. The same machinery might therefore be expected to diversify functionally in complex metazoan organisms, so as to regulate new layers of defences used by multicellular organisms to confront different forms of stress. A plethora of genetic, biochemistry, cell biology, systems biology and genomic studies have recently converged to support this notion. The autophagy machinery interfaces with most cellular stress-response pathways ${ }^{3}$, including those involved in controlling immune responses and inflammation. This interface is not only at the level of the autophagy pathway, but also entails direct interactions between autophagy proteins and immune signalling molecules ${ }^{4}$. There is a complex reciprocal relationship between the autophagy pathway/proteins and immunity and inflammation; the autophagy proteins function in both the induction and suppression of immune and inflammatory responses, and immune and inflammatory signals function in both the induction and suppression of autophagy. Moreover, similar to cancer, neurodegenerative diseases and ageing ${ }^{5}$, defects in autophagy — through autophagy gene mutation and/or microbial antagonism - may underlie the pathogenesis of many infectious diseases and inflammatory syndromes.

In this Review, we describe recent advances in our evolving comprehension of the interface between autophagy, immunity and inflammation. We discuss how emerging concepts about the functions of the autophagy pathway and the autophagy proteins may reshape our understanding of immunity and disease. This set of proteins not only orchestrates the lysosomal degradation of unwanted cargo, but also exerts intricate effects on the control of immunity and inflammation. Thus, the autophagy pathway and autophagy proteins may function as a central fulcrum that balances the beneficial and harmful effects of the host response to infection and other immunological stimuli.

\section{Mechanisms and membrane dynamics of autophagy}

Autophagy is a general term for pathways by which cytoplasmic material, including soluble macromolecules and organelles, is delivered to lysosomes for degradation ${ }^{6}$. There are at least three different types of autophagy, including macroautophagy, chaperone-mediated autophagy and microautophagy. Macroautophagy, usually referred to simply as autophagy, is the subject of this Review (Fig. 1). In this pathway, a portion of cytoplasm (usually $0.5-1 \mu \mathrm{m}$ in diameter) is engulfed by an isolation membrane, or 'phagophore', resulting in the formation of a double-membrane structure known as the autophagosome. The outer membrane of the autophagosome fuses with the lysosome to become an autolysosome, leading to the degradation of autophagosomal contents by lysosomal enzymes. Autophagosomes can also fuse with endosomes or multivesicular bodies and major histocompatibility complex (MHC)class-II-loading compartments ${ }^{7}$. Autolysosomes become larger as more autophagosomes and lysosomes fuse, but at a termination phase lysosomes are tubulated and fragmented for renewal ${ }^{8}$.

The membrane dynamics of autophagosome formation involve complex processes, which are not completely understood. Nonetheless, the molecular dissection of autophagy membrane dynamics, stimulated by the discovery of $A T G$ (autophagy-related) genes in yeast ${ }^{9}$, has shed considerable light on this topic (Table 1). Several recent studies suggest that the endoplasmic reticulum (ER) is crucial for autophagosome formation. The ER cisternae often associate with developing autophagosomes, and electron tomography analysis has demonstrated direct connections between the ER and autophagosomal membranes ${ }^{10,11}$. Moreover, the function of several key autophagy proteins seems to be at the level of the ER (Fig. 1).

Autophagy is induced by nutrient starvation through the inhibition

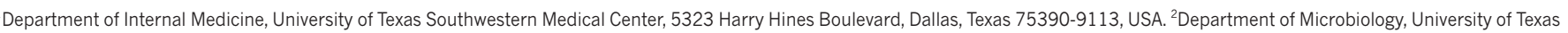

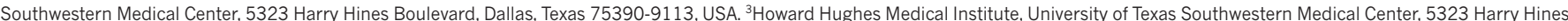

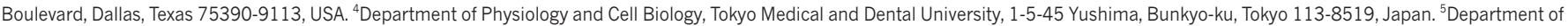
Pathology and Immunology, Washington University School of Medicine and Midwest Regional Center of Excellence for Biodefense and Emerging Infectious Diseases Research, Campus Box 8118 , 660 South Euclid Avenue, Saint Louis, Missouri 63110, USA. 


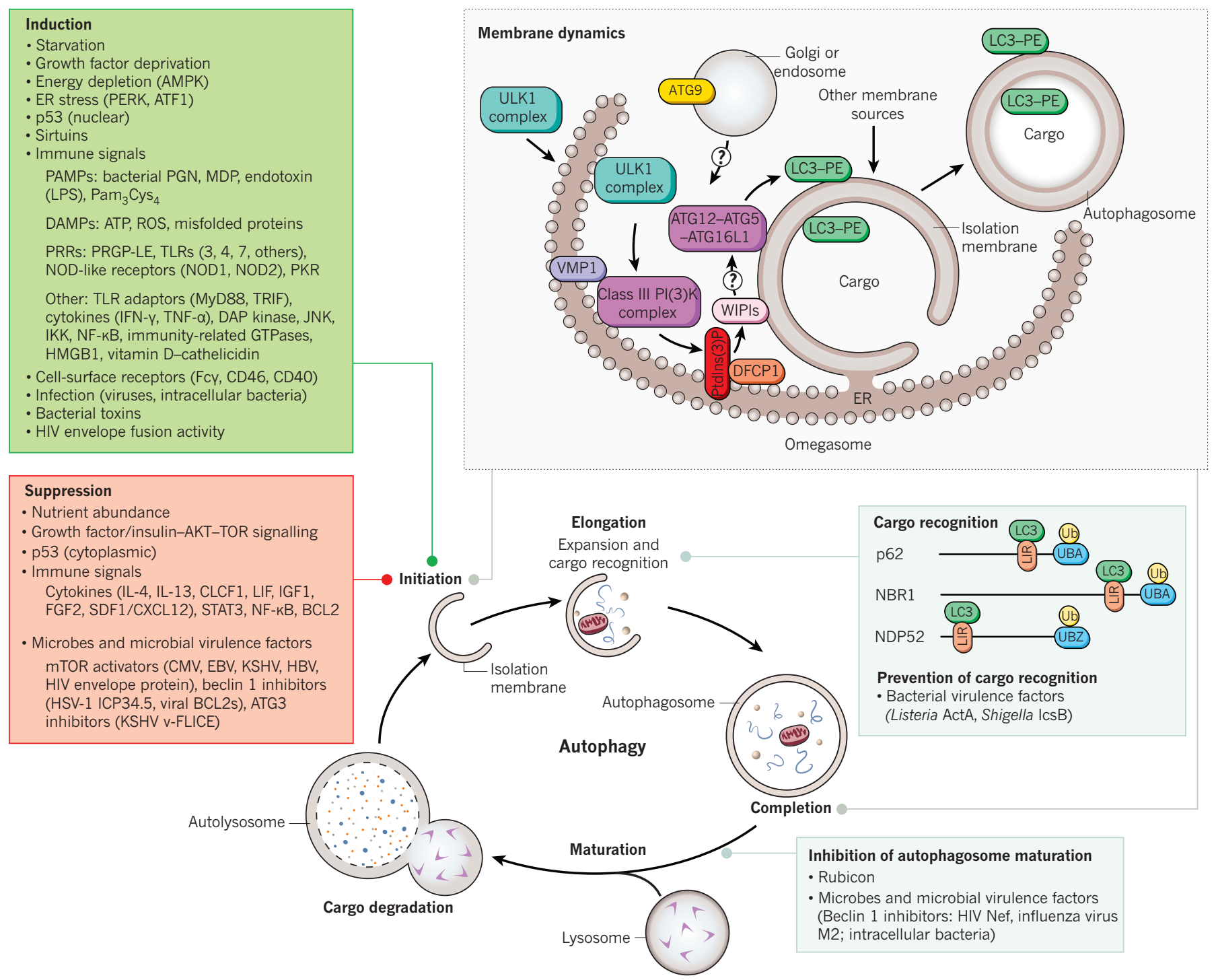

Figure 1 | Schematic overview of autophagy and its regulation. Overview of the autophagy pathway. The top right box shows a model of our current understanding of the molecular events involved in membrane initiation, elongation and completion of the autophagosome. The major membrane source is thought to be the endoplasmic reticulum (ER), although several other membrane sources, such as mitochondria and the plasma or nuclear membrane, may contribute. After induction of autophagy, the ULK1 complex (ULK1-ATG13-FIP200-ATG101) (downstream of the inhibitory mTOR signalling complex) translocates to the ER and transiently associates with VMP1, resulting in activation of the ER-localized autophagy-specific class III phosphatidylinositol-3-OH kinase $(\mathrm{PI}(3) \mathrm{K})$ complex, and the phosphatidylinositol-3-phosphate (PtdIns(3)P) formed on the ER membrane recruits DFCP1 and WIPIs. WIPIs and the ATG12-ATG5-ATG16L1 complex are present on the outer membrane, and LC3-PE is present on both the outer and inner membrane of the isolation membrane, which may emerge from subdomains of the ER

of mammalian target of rapamycin (mTOR), resulting in translocation of the mTOR substrate complex (ULK1/2, ATG13, FIP200 (also known as RB1CC1) and ATG101) from the cytosol to certain domains of the ER or closely attached structures ${ }^{12,13}$. This leads to the recruitment of the class III phosphatidylinositol-3-OH kinase (PI(3)K) complex, which includes at least VPS34 (also known as PIK3C3), VPS15 (PIK3R4 and p150), beclin 1 and ATG14, to the $\mathrm{ER}^{13,14}$. The PI(3)K complex produces phosphatidylinositol-3-phosphate (PtdIns(3)P), which recruits effectors such as double FYVE-containing protein 1 (DFCP1) and WDrepeat domain phosphoinositide-interacting (WIPI) family proteins. termed omegasomes. The cellular events that occur during autophagy are depicted in the bottom diagram, including the major known cellular and microbial proteins that regulate autophagy initiation, cargo recognition and autophagosome maturation. Only those cellular proteins known to be adaptors for targeting microbes are shown; other proteins (not shown) also function in cargo recognition of mitochondria and other organelles. CMV, cytomegalovirus; DAMP, danger-associated molecular pattern; DAP, death-associated protein; EBV, Epstein-Barr virus; HBV, hepatitis B virus; HSV-1, herpes simplex virus 1; KSHV, Kaposi's sarcoma-associated herpesvirus; LIR, LC3-interacting region (motif); LPS, lipopolysaccharide; MDP, muramyl dipeptide; $\mathrm{Pam}_{3} \mathrm{Cys}_{4}$, a synthetic TLR2 agonist; PAMP, pathogen-associated molecular pattern; PERK, an eIF2a kinase; PGN, peptidoglycan; PRGP-LE, a peptidoglycan-recognition protein; PRR, pathogen-recognition receptor; ROS, reactive oxygen species; Ub, ubiquitin; UBA, ubiquitin-associated domain; UBZ, ubiquitin-binding zinc finger; v-FLICE, viral FLICE.

DFCP1 is diffusely present on the ER or the Golgi, but translocates to the autophagosome formation site in a PtdIns(3)P-dependent manner to generate ER-associated $\Omega$-like structures termed omegasomes ${ }^{15}$. Among the four WIPI isoforms, WIPI2 is the major form in most cell types and functions downstream of DFCP1, and it may promote the development of omegasomes into isolation membranes or autophagosomes ${ }^{16}$.

An ER-associated multispanning membrane protein, VMP1, is also important for autophagosome formation. Although VMP1 interacts with beclin 1 and is present at the autophagosome formation site at an early stage, it seems to function at a late stage in autophagy ${ }^{13,17,18}$. 
Table 1 | Key proteins involved in mammalian autophagosome formation and their immune functions

\begin{tabular}{|c|c|c|c|c|}
\hline $\begin{array}{l}\text { Protein } \\
\text { complex }\end{array}$ & Function of protein complex in autophagy & Specific protein & General properties & Immunological/host defence functions \\
\hline \multicolumn{5}{|c|}{ Nucleation step of autophagosome formation } \\
\hline \multirow[t]{4}{*}{$\begin{array}{l}\text { ULK } \\
\text { complex }\end{array}$} & \multirow{4}{*}{$\begin{array}{l}\text { This complex is negatively regulated } \\
\text { by mTORC1 in a nutrient-dependent } \\
\text { manner. After autophagy induction, } \\
\text { this complex translocates to early } \\
\text { autophagic structures. Although } \\
\text { FIP200 and ATG13 are known } \\
\text { to be phosphorylated by ULK1, } \\
\text { physiologically relevant substrates } \\
\text { remain unknown. FIP200 and } \\
\text { ATG101 may have functions similar } \\
\text { to yeast Atg17, } 29 \text { and } 31 \text {, although } \\
\text { they show no sequence similarity } \\
\text { with these proteins. }\end{array}$} & ULK1/2 & $\begin{array}{l}\text { Protein kinase, } \\
\text { phosphorylated by mTORC1 }\end{array}$ & Antibacterial ${ }^{47 *, 48 *}$; antiviral ${ }^{46 *}$ \\
\hline & & ATG13 & Phosphorylated by mTORC1 & Unknown \\
\hline & & FIP200 & $\begin{array}{l}\text { Scaffold for ULK1/2 and } \\
\text { ATG13 }\end{array}$ & Maintains numbers of fetal haematopoietic stem cells ${ }^{72}$ \\
\hline & & ATG101 & Interacts with ATG13 & Unknown \\
\hline \multirow{7}{*}{$\begin{array}{l}\text { Class III } \\
\mathrm{PI}(3) \mathrm{K} \\
\text { complex }\end{array}$} & \multirow{7}{*}{$\begin{array}{l}\text { Beclin } 1 \text { is negatively regulated } \\
\text { by BCL2 and by BCL-X through } \\
\text { direct binding. This complex } \\
\text { produces Ptdlns(3)P, probably on } \\
\text { the ER. VPS34, VPS15 and beclin } \\
1 \text { are shared with the UVRAG } \\
\text { complex, which seems to function } \\
\text { in the late endocytic pathway. } \\
\text { Rubicon negatively regulates } \\
\text { autophagosome-lysosome fusion } \\
\text { through interaction with the UVRAG } \\
\text { complex. }\end{array}$} & VPS34 & $\mathrm{PI}(3)$ kinase & Antiviral $^{45 *}$; phagosome maturation ${ }^{33}$ \\
\hline & & VPS15 & Myristoylated & Unknown \\
\hline & & Beclin 1 & $\begin{array}{l}\text { BH3-only protein, interacts } \\
\text { with BCL2 and BCL- } X_{L}\end{array}$ & $\begin{array}{l}\text { Antibacterial }{ }^{45 *, 48 *} \text {; antiviral }{ }^{46 *, 57,58} \text {; apoptotic corpse } \\
\text { clearance }^{84} \text {; decreases inflammation in tumours }{ }^{5} \text {; regulates } \\
\text { germinal centre induction }{ }^{5} \text {; phagosome maturation }{ }^{31,33,49} \text {; } \\
\text { interacts with TLR signalling adaptors }\end{array}$ \\
\hline & & ATG14 & Autophagy-specific subunit & Unknown \\
\hline & & AMBRA1 & $\begin{array}{l}\text { Interacts with and activates } \\
\text { beclin } 1\end{array}$ & Unknown \\
\hline & & UVRAG & $\begin{array}{l}\text { A VPS38 homologue; } \\
\text { interacts with class C VPS } \\
\text { (HOPS) complex }\end{array}$ & Unknown \\
\hline & & Rubicon & Interacts with beclin 1 & Unknown \\
\hline \multirow[t]{5}{*}{ Others } & \multirow{5}{*}{$\begin{array}{l}\text { DFCP1 forms an omegasome on } \\
\text { the ER, at which other ATG proteins } \\
\text { are assembled. ATG9, WIPIs and } \\
\text { VMP1 are present on the autophagic } \\
\text { membrane. ATG9 also exists in other } \\
\text { compartments such as endosomes } \\
\text { and the Golgi apparatus. }\end{array}$} & ATG2 & Interacts with Atg18 in yeast & Antiviral $^{46 *}$ \\
\hline & & ATG9 & Transmembrane protein & Antiviral ${ }^{46 *}$; inhibits innate immune signalling ${ }^{4}$ \\
\hline & & WIPI1-4 & Ptdlns(3)P-binding proteins & Unknown \\
\hline & & DFCP1 & Ptdlns(3)P-binding ER protein & Unknown \\
\hline & & VMP1 & Transmembrane protein & Unknown \\
\hline
\end{tabular}

\section{Elongation step}

ATG12- The ATG12-ATG5-ATG16L1 dimer is ATG12

conjugation important for LC3-PE conjugation.

system This complex is present on the outer side of the isolation membrane and is essential for proper elongation of the isolation membrane.
Ubiquitin-like, conjugates to ATG5

ATG7 E1-like enzyme

ATG10 E2-like enzyme

ATG5 Conjugated by ATG12

ATG16L1 Homodimer, interacts with

LC3- The formation of LC3-PE

conjugation conjugates and their

system important for isolation membrane elongation and/or complete closure. LC3 is present on both the inner and outer membrane of the autophagosomes, and also serves as an adaptor for selective substrates such as p62, NBR1, NDP52 and the yeast mitophagy protein Atg32. ATG5

$\begin{array}{ll} & \text { ATG5 } \\ \text { LC3 } & \text { Ubiquitin-like, conjugates } \\ \text { (GATE16, } & \text { to PE } \\ \text { ATABARAP) } & \\ & \begin{array}{l}\text { LC3 carboxy-terminal } \\ \text { hydrolase, deconjugating } \\ \text { enzyme }\end{array} \\ \text { ATG7 } & \text { E1-like enzyme }\end{array}$

\section{ATG7 E1-like enzyme}

\author{
Antiviral $^{46 *}$; antibacterial ${ }^{34}$; antigen presentation ${ }^{7,32,74}$; inhibits \\ type I IFN production ${ }^{78}$ \\ Antiviral $^{45 *, 46 *}$; antibacterial ${ }^{48 *}$; antigen presentation ${ }^{32}$; \\ phagosome maturation ${ }^{31}$; maintains number of T cells ${ }^{44,72}$; \\ intestinal immune epithelial cell function ${ }^{90}$; inhibits type I IFN \\ production ${ }^{78}$; inhibits pro-inflammatory cytokine production ${ }^{82}$ \\ Unknown
}

Antiviral $^{40,46 *}$; antibacterial ${ }^{1,30,35,47 *, 100}$; antiparasitic ${ }^{35}$; antigen presentation $^{27,32,73}$; phagosome maturation ${ }^{31}$; apoptotic corpse clearance ${ }^{84}$; maintains number of $\mathrm{T}$ cells $\mathrm{s}^{44,72}$; maintains number of B1a B cells ${ }^{44}$; intestinal immune epithelial cell function ${ }^{90}$; inhibits type I IFN production ${ }^{77,78}$; increases type I IFN production ${ }^{76}$

Antibacteria| ${ }^{87,88}$; antigen presentation ${ }^{52}$; Crohn's disease risk allele ${ }^{85}$; intestinal immune epithelial cell function ${ }^{81,90}$; inhibits pro-inflammatory cytokine production ${ }^{82}$

Antiviral $^{46 *}$; antibacterial| ${ }^{48 *}$; antigen presentation ${ }^{7}$; adaptor for substrates of selective autophagy of microbes (xenophagy) $^{38,41,61}$

Antiviral ${ }^{46 *}$

Antiviral ${ }^{45 *, 46 *}$; antibacterial ${ }^{48 *}$; antigen presentation ${ }^{32}$; phagosome maturation ${ }^{31}$; maintains number of T cells ${ }^{44,72}$; intestinal immune epithelial cell function ${ }^{90}$; inhibits type I IFN production $^{78}$; inhibits pro-inflammatory cytokine production ${ }^{82}$

Antiviral ${ }^{45 *}$

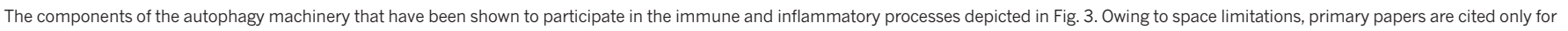
those citations also included in the main text; otherwise, see references contained within cited review articles.

*Function observed in model organism (for example, Dictyostelium discoideum, Nicotiana benthamiana, Arabidopsis thaliana, Drosophila melanogaster or Caenorhabditis elegans). 
This is perhaps consistent with other evidence that beclin 1-class III $\mathrm{PI}(3) \mathrm{K}$ complexes may function in autophagosomal maturation (in addition to vesicle nucleation), a process that can be regulated by other beclin-1-interacting partners such as rubicon (Table 1). At the final step of autophagosome formation, elongation of the isolation membrane and/or completion of enclosure require two ubiquitin-like conjugates. The first is the ATG12-ATG5 conjugate, which is produced by the ATG7 (E1-like) and ATG10 (E2-like) enzymes, and functions as a dimeric complex together with ATG16L1 (ref. 19). The second is the phosphatidylethanolamine (PE)-conjugated ATG8 homologues LC3, GATE16 and GABARAP - which are produced by the ATG7 and ATG3 (E2-like) enzymes ${ }^{9,20}$. Although the proteins involved in autophagosome membrane formation have been characterized as discrete complexes (Table 1), several potential interconnections between components of the different complexes were identified by a recent proteomics study ${ }^{21}$. Such interconnections may function in autophagosome membrane formation or other distinct cellular functions. For example, the ATG12-ATG3 conjugate is implicated in mitochondrial homeostasis but not in autophagosome membrane formation ${ }^{22}$.

In addition to the ER, other membranes may be involved in autophagosome formation (Fig. 1). ATG9, another multispanning membrane protein, is essential for autophagy ${ }^{23}$ and traffics between the trans-Golgi network, endosomes and autophagosome precursors ${ }^{24}$. Studies suggest that mitochondria, the plasma membrane and the nuclear membrane could also be membrane sources for autophagosome formation ${ }^{25-27}$. However, the lack of detection of specific protein markers for these structures on the autophagosomal membrane leaves the decades-old question of the membrane source of the autophagosome unanswered. It is possible that cells may use different membrane sources to form the autophagosome in different contexts, thereby permitting specialization of membrane dynamics in a manner that allows divergent autophagyinducing signals to stimulate the capture of spatially distinct cargo.

\section{Selective autophagy tackles microbes}

Autophagy was originally considered to be a non-selective bulk degradation process, but it is now clear that autophagosomes can degrade substrates in a selective manner ${ }^{28}$. In addition to endogenous substrates, autophagy degrades intracellular pathogens in a selective form of autophagy, termed xenophagy. Similar to bulk autophagy (such as that induced by nutrient deprivation) and other forms of selective autophagy (such as degradation of damaged mitochondria, peroxisomes, aggregate-prone proteins or damaged ER), the precise membrane dynamics and specificity determinants of xenophagy are not fully understood. Nonetheless, considerable advances have been made, and interesting similarities and differences are beginning to emerge between cellular recognition and degradation of self versus foreign microbial components through autophagy-like pathways (Figs 1 and 2).

The vacuoles used for the engulfment of intracytoplasmic bacteria are similar to autophagosomes, and their formation requires the core autophagy machinery. But one apparent difference is the vacuole size; for example, the diameter of group A Streptococcus-containing autophagosome-like vacuoles (GcAV) can be as big as $10 \mu \mathrm{m}$. These large GcAVs are generated by the RAB7-dependent fusion of small isolation membranes ${ }^{29}$. By contrast, the formation of starvation-induced autophagosomes requires RAB7 later in the autophagy process, at the autophagosome-lysosome fusion step.

A more complex question is how autophagosomes (or components of the autophagy pathway) capture pathogens that are inside vacuolar compartments (Fig. 2). There are at least four general pathways that may be used for autophagy-protein-dependent targeting of bacteria to the lysosome. These include autophagy-protein-facilitated fusion of bacteria-containing phagosomes with lysosomes, the envelopment of bacteria-containing phagosomes or endosomes by autophagosomal membranes, the fusion of bacteria-containing phagosomes or endosomes with autophagosomes, or the xenophagic capture of bacteria that have escaped inside the cytoplasm. In some cases, the route of autophagy-dependent targeting to the lysosome has been well defined, such as for group A Streptococcus that escapes from endosomes $^{30}$. For several bacteria, however, the precise route is unclear. Many studies define bacterial autophagy as the co-localization of bacteria and LC3, but we now know that LC3 can decorate membranous compartments other than autophagosomes (including phagosomes).

Several lines of evidence suggest that autophagy proteins function more broadly, not only in classical macroautophagy, but also in the process of phagolysosomal maturation during antigen presentation and microbial invasion. Autophagy proteins are required for the fusion of phagosomes that contain Toll-like receptor (TLR)-ligand-enveloped particles with lysosomes in macrophages ${ }^{31}$, and for the fusion of phagosomes that contain TLR-agonist-associated apoptotic cell antigens with lysosomes in dendritic cells during MHC class II antigen presentation ${ }^{32}$. The self ligand and cell-surface receptor SLAM functions as a microbial sensor that recruits the beclin 1-class III PI(3)K complex to phagosomes containing Gram-negative bacteria, facilitating phagolysosomal fusion and activation of the antibacterial NADPH oxidase (NOX2) complex ${ }^{33}$. In addition, the engagement of TLR or $\mathrm{Fc} \gamma$ receptors during phagocytosis recruits LC3 (and ATG12) to the phagosome through NOX2dependent generation of reactive oxygen species (ROS) ${ }^{34}$. Thus, in bacterial infections, a paradigm is emerging in which the coordinated regulation of microbial sensing, phagolysosomal maturation and antibacterial activity involves the recruitment of autophagy proteins to the phagosome. As a corollary, an interesting speculation is that impaired recruitment of autophagy proteins to the phagosome may contribute to the pathogenesis of chronic granulomatous disease, a genetic disorder caused by mutations in the NOX2 gene (also known as CYBB) and characterized by recurrent bacterial and fungal infections and inflammatory complications, such as inflammatory bowel disease.

Another autophagosome-independent function of autophagy proteins in pathogen destruction has been described in interferon- $\gamma$ (IFN- $\gamma$ )treated macrophages infected with the parasite Toxoplasma gondii. The parasite-derived membrane, termed the parasitophorous vacuole, undergoes destruction through a mechanism that involves ATG5-dependent recruitment of the immunity-related GTPase proteins to the parasitophorous vacuole ${ }^{35,36}$, leading to the death of the parasite in the infected cell ${ }^{35,37}$ Together, these studies suggest that autophagy proteins have diverse roles in membrane dynamics to benefit the host in the removal of invading pathogens (Fig. 2), through xenophagy, phagolysosomal maturation, the recruitment of molecules that damage pathogen-derived membranes, and presumably, many other as yet undiscovered mechanisms.

The mechanisms that cells use to target intracellular bacteria (and probably viruses) to autophagosomal compartments are notably similar to those used for selective autophagy of endogenous cargo. Cellular cargo is commonly targeted to autophagosomes by interactions between a molecular tag (such as polyubiquitin), adaptor proteins such as p62 (also known as SQSTM1 or sequestome 1) or NBR1 (which recognize these tags and contain an LC3-interacting region (LIR) characterized by a WXXL or WXXI motif), and LC3 (ref. 28). These adaptor molecules enable autophagy to target designated cargo selectively to nascent LC3-positive isolation membranes. As reviewed elsewhere ${ }^{38}$, a similar mechanism involving ubiquitin and 162 seems to be involved in the targeting of intracellular bacteria, such as Salmonella enterica serotype Typhimurium (S. Typhimurium), Shigella flexneri and Listeria monocytogenes, to autophagosomes.

After escape into the cytoplasm or in vacuolar membrane compartments damaged by type III secretion system (T3SS) effectors, bacteria or bacteria-containing compartments, respectively, may become coated with ubiquitin and associate with p62 and nascent LC3-positive isolation membranes. The autophagosomal targeting of Salmonella also requires another cellular factor, NDP52 (nuclear dot protein 52), an autophagy adaptor protein that, like p62, contains an LIR and ubiquitin-binding domains and restricts intracellular bacterial replication. A ubiquitinindependent pathway (that does not involve p62 or NDP52) could also function in targeting damaged Salmonella-containing vacuoles (SCVs) 


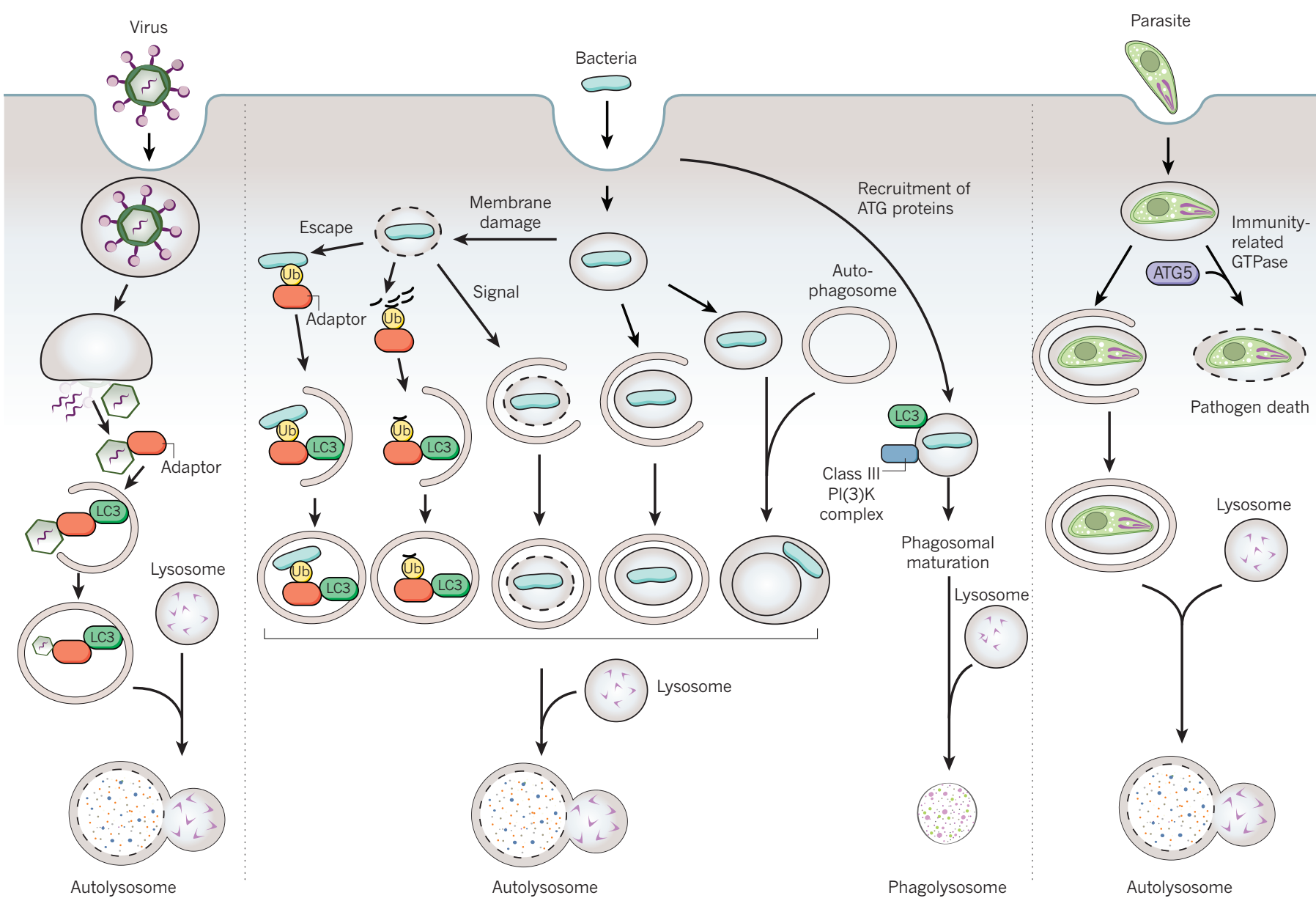

Figure 2 | Possible autophagy-protein-dependent pathways of pathogen degradation. Possible pathways involving the autophagy machinery by which viruses, bacteria (and damaged membranes of bacteria-containing vacuoles) and parasites may be targeted to the lysosome. Adaptor refers

to the autophagosome. In this pathway, a lipid second messenger, diacylglycerol, acts as a signal for the co-localization of SCVs with LC3-positive autophagosomes by a mechanism that involves protein kinase $\mathrm{C}$ and its downstream targets, JNK and NADPH oxidase ${ }^{39}$. The autophagic targeting of a cytoplasmic positive-strand RNA virus, Sindbis virus, also occurs in a ubiquitin-independent manner, but involves the interaction of p62 with the viral capsid protein ${ }^{40}$. Thus, diverse molecular strategies, including ubiquitin-dependent and -independent mechanisms, may be used to target microbes inside the cytoplasm or vacuolar compartments to the autophagosome.

Beyond targeting intracellular pathogens for degradation, p62 may have further beneficial effects in infected host cells. For example, Shigella vacuolar membrane remnants generated by bacterial T3SS-dependent membrane damage are targeted by polyubiquitination, p62 and LC3 for autophagosomal degradation ${ }^{41}$ (Fig. 2). These membrane remnants also accumulate numerous molecules involved in sensing and transduction of pathogen-associated molecular pattern (PAMP) and danger-associated molecular pattern (DAMP) signals, and there is an increase in nuclear factor- $\kappa \mathrm{B}(\mathrm{NF}-\kappa \mathrm{B})$-dependent cytokine production, ROS production and necrotic cell death in autophagy-deficient cells. Thus, the ubiquitin-p62-dependent autophagic targeting of pathogen-damaged membranes could help to control detrimental downstream inflammatory signalling during bacterial invasion into host cells. Another emerging concept is that selective autophagy of viral proteins, similar to selective autophagy of aggregate-prone toxic cellular proteins, may protect post-mitotic cells such as neurons against cell death. For example, in Sindbis-virus-infected mice with neuron-specific inactivation to the proteins shown in the cargo-recognition box in Fig. 1; however, as yet undiscovered adaptors may be involved in pathogen recognition, and pathogen targeting may involve ubiquitin-dependent or -independent mechanisms.

of Atg5, there is an accumulation of Sindbis virus antigens (without increased levels of infectious virus), increased neuronal cell death and increased animal mortality ${ }^{40}$. Moreover, p62 is required for starvation and IFN- $\gamma$-induced targeting of Fau (and perhaps other ubiquitylated protein complexes) to mycobacteria-containing phagosomes, resulting in the generation of antimycobacterial Fau-derived peptides ${ }^{42}$. The role of p62 in innate immunity is probably evolutionarily ancient, as the Drosophila 662 orthologue REF(2)P was originally identified in a screen for modifiers of sigma virus replication ${ }^{43}$.

We speculate that p62, as well as the other known LC3-interacting adaptor proteins (NBR1 and NDP52), may represent the tip of the iceberg in terms of cellular adaptor proteins that bind to ubiquitin (or other molecular tags) and target microbial substrates and cytosolic material to autophagosomes to coordinate innate immune responses. A recent proteomics study showed that the mammalian ATG8 family, which includes LC3, GATE16 and GABARAP, has 67 high-confidence interactions with other cellular proteins ${ }^{21}$. Some of these new ATG8-familymember-interacting partners may have an as yet undiscovered role in innate immunity. Another open question is whether the known proteins involved in selective autophagy of mitochondria (called mitophagy), such as Nix (also known as BNIP3L) and parkin ${ }^{44}$, also function in microbial autophagy.

\section{Autophagy and resistance to infection}

The autophagy pathway and/or autophagy proteins have a crucial role in resistance to bacterial, viral and protozoan infection in metazoan organisms. The genetic deletion or knockdown of autophagy genes 
protects plants from viral, fungal and bacterial infection by preventing the uncontrolled spread of programmed cell death during the plant innate immune or hypersensitive response ${ }^{45}$. In other organisms, autophagy proteins function in a cell-autonomous manner to control infection by intracellular pathogens. In Drosophila, autophagy gene mutation increases susceptibility to viral (vesicular stomatitis virus) ${ }^{46}$ and bacterial (L. monocytogenes) ${ }^{47}$ infection. In Dictyostelium and Caenorhabditis elegans, autophagy gene mutation increases susceptibility to lethal $S$. Typhimurium infection ${ }^{48}$. In mice, knockout of Atg5 in macrophages and neutrophils increases susceptibility to infection with $L$. monocytogenes and the protozoan T. gondii ${ }^{35}$, and neuron-specific Atg 5 knockout increases susceptibility to central nervous system Sindbis virus infection ${ }^{40}$. As noted in the next section, the autophagy pathway and proteins may also have 'proviral' or 'probacterial' effects in in vitro studies; however, in vivo evidence for such effects is so far lacking. The mechanisms by which autophagy genes mediate in vivo resistance to infection are not fully understood, but are likely to involve a combination of xenophagy, other autophagy-protein-dependent effects on microbial replication or survival, activation of innate and adaptive immune responses, and/or alterations in pathogen-induced cell death (Fig. 3).

An important question is whether this function of autophagy in broad resistance to infection with intracellular pathogens extends to humans. Recent human genetic studies provide some clues. The immunityrelated GTPase human IRGM, which regulates autophagy-dependent clearance of mycobacteria in vitro ${ }^{49}$, was identified as a genetic risk locus for tuberculosis in a West African population ${ }^{50}$. Numerous studies have shown a crucial role for autophagy in defence against mycobacterial infection in human cells ${ }^{1}$, and a genome-wide analysis of host genes that regulate Mycobacterium tuberculosis replication demonstrated that a predominance of factors were autophagy regulators ${ }^{51}$. Thus, it is possible that autophagy has a central role in resistance to one of the most important global infectious diseases - tuberculosis. Mutations in NOD2, which encodes an intracellular pathogen-recognition receptor (nucleotide-binding oligomerization-domain-containing protein 2) that functions in bacterial autophagy $y^{52,53}$, are also associated with susceptibility to infection with another mycobacterial agent, Mycobacterium leprae, the aetiological agent of leprosy ${ }^{54}$. An exciting future venture will be to confirm whether IRGM, NOD2 and other autophagy-related genes are involved in resistance to infection with mycobacteria and other infections in further human populations and, if so, whether this resistance is mediated by autophagy.

\section{Microbes fight back}

Microbes undergo strong selective pressure to develop strategies to block host defence mechanisms; the number of such strategies is a surrogate measure of the importance of the host defence mechanism in immunity. As reviewed elsewhere ${ }^{1,55}$, viruses and intracellular bacteria have evolved several ways to adapt to host autophagy. They can antagonize autophagy initiation or autophagosomal maturation, evade autophagic recognition, or use components of the autophagy pathway to facilitate their own replication or intracellular survival. An emerging theme is that microbial antagonism of autophagy not only blocks the xenophagic degradation of intracellular pathogens, but also blocks the functions of autophagy in innate and adaptive immunity. A relatively unexplored yet crucially important frontier is how microbial antagonism may contribute more broadly to the role of microbes in diseases characterized by defective autophagy, such as cancer, neurodegenerative diseases, ageing and, potentially, autoimmune and inflammatory diseases.

Viral strategies to shut off autophagy include the blockade of positive upstream regulators of autophagy (such as the IFN-inducible RNA-activated eIF2 $\alpha$ protein kinase (PKR) signalling pathway), the activation of negative upstream regulators of autophagy (such as the nutrient-sensing TOR kinase signalling pathway) or direct antagonism of the autophagy machinery ${ }^{55}$. The overlapping functions of the eIF2 $a$ kinase signalling pathway in stress-induced general translational arrest,

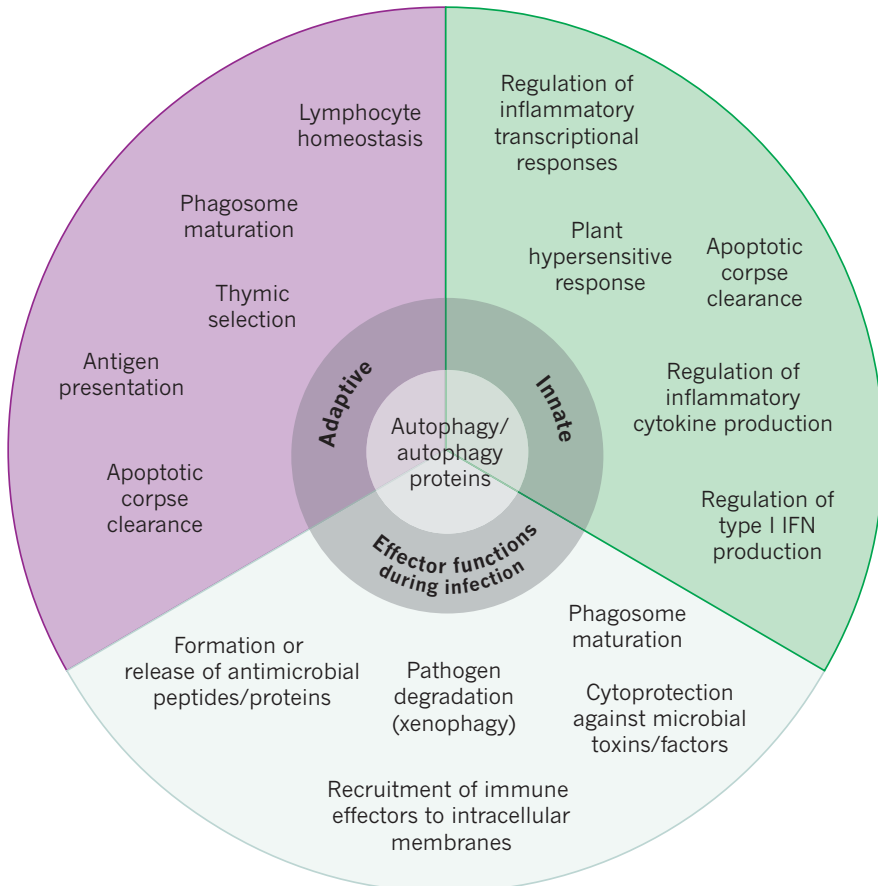

Figure 3 | Functions of the autophagy pathway and/or proteins in immunity. A summary of the known functions of the autophagy pathway and/or proteins in adaptive and innate immunity, and as effectors during infection.

transcriptional activation of stress-response genes and stress-induced autophagy enable viruses to disarm several facets of the cellular stress response to infection by one mechanism - that is, antagonism of eIF2 $\alpha$ kinase signalling. The mTOR signalling pathway has a central role in the control of cell growth and metabolism, and interestingly, many of the viruses that activate mTOR are oncogenic (for example, Epstein-Barr virus, Kaposi's sarcoma-associated herpesvirus, hepatitis B virus and retroviruses). This suggests another type of pluripotent viral weapon - one that can promote oncogenesis by simultaneously inactivating autophagy and promoting cell growth through TOR activation. HIV envelope protein-dependent activation of mTOR signalling is also proposed to be a mechanism for HIV evasion of innate and adaptive immune responses in dendritic cells, including the degradation of incoming virions by lysosomes, blockade of HIV transfer to $\mathrm{CD} 4^{+} \mathrm{T}$ cells, stimulation of TLR4 and TLR8 ligand responses, and presentation of HIV Gag antigen to CD $4^{+} \mathrm{T}_{\text {cells }}{ }^{56}$. It will be important to determine whether these effects of HIV-mediated mTOR activation and autophagy inhibition contribute to impaired dendritic cell function during HIV infection in vivo.

Several viral proteins target the core autophagy protein beclin 1 . Autophagosome initiation is blocked by interactions between beclin 1 and the herpes simplex virus 1 (HSV-1) neurovirulence factor ICP34.5 or the oncogenic $\gamma$-herpesvirus-encoded viral BCL2-like proteins, whereas autophagosome maturation is blocked by interactions between beclin 1 and the HIV accessory protein Nef or the influenza virus matrix protein 2 (ref. 55). The interactions between beclin 1, HSV-1 ICP34.5 and viral BCL2 are probably physiologically important in vivo; a mutant HSV-1 virus lacking the beclin-1-binding domain of ICP34.5 is attenuated in mouse models of encephalitis (presumably through its failure to control xenophagy and innate immunity $)^{57}$ and of corneal disease (through its failure to control adaptive immunity $)^{58}$. Moreover, a mouse $\gamma$-herpesvirus that encodes a mutant viral BCL2 unable to bind to beclin 1 demonstrates impaired ability to maintain chronic infection ${ }^{59}$. Thus, viral antagonism of host autophagy can manipulate distinct aspects of viral pathogenesis and immunity in vivo.

It is not yet clear whether compared with other autophagy proteins, 
beclin 1 is preferentially targeted by viral virulence proteins because of its central role in autophagosome formation, or more likely, whether we are just beginning to identify pairs of viral proteins and their autophagy pathway targets. In support of the latter, viral FLICE-like inhibitors encoded by Kaposi's sarcoma-associated herpesvirus and molluscum contagiosum virus suppress autophagy by interacting with the ATG3 E2-like enzyme, thereby preventing it from binding and processing LC3 (ref. 60).

Bacteria possess diverse strategies to avoid degradation by autophagolysosomal pathways. As reviewed elsewhere ${ }^{1,38}$, many bacteria that reside in phagosomes or other vacuolar compartments have methods to inhibit lysosomal fusion or maturation, which, in the case of mycobacteria, can be partially overcome by treatments that stimulate autophagy. Another possible mechanism for bacterial evasion of autophagy has emerged from a genome-wide screen to identify host factors that regulate the intracellular survival of $M$. tuberculosis ${ }^{51}$. According to bioinformatics analyses, $M$. tuberculosis infection of human macrophage-like cells activates cellular pathways that inhibit autophagy. Intracellular bacteria that escape into the cytoplasm, such as S. flexneri and L. monocytogenes, use strategies to camouflage themselves to avoid autophagic recognition. The Shigella T3SS effector IcsB competitively binds to VirG, thereby preventing the interaction between ATG5 and VirG, a bacterial surface protein required for actin-based motility and Shigella targeting to autophagosomes ${ }^{38}$. The Listeria protein ActA interacts with cytosolic actin polymerization machinery (ARP2/3, VASP and actin), which blocks bacterial association with ubiquitin, p62 recruitment and autophagic targeting ${ }^{61}$. The precise mechanism of this block is unknown, but it has been proposed that the ActA-dependent recruitment of host cell cytoskeletal proteins may enable the bacterium to disguise itself as a host cell organelle ${ }^{61}$. This concept sheds light on the autophagy pathway in a fundamental aspect of immunology - the basis for discrimination between self and non-self.

Microbes have evolved not only to antagonize autophagy (as a cellular defence mechanism that threatens their survival), but also to exploit its components and functions in membrane trafficking for their own self-serving purposes ${ }^{1,55}$. An early concept in the field is that autophagosomes may serve as a protected niche for intracellular bacteria (provided fusion with acidic compartments is blocked) and/or serve as a source of nutrients for intracellular pathogens (which would require intact autophagolysosomal fusion $)^{1}$. Trafficking of the intracellular bacteria Yersinia pseudotuberculosis to acidic compartments was recently shown to be enhanced by genetic inhibition of autophagy ${ }^{62}$. This seemingly contradicts other evidence that the autophagy proteins promote phagosomal maturation, but is consistent with the concept that autophagosomes function as a protected intracellular niche for bacteria. The role of the autophagy machinery in promoting and/or inhibiting vacuolar acidification - and the counter effects of microbes that reside in vacuolar compartments - needs to be explored further.

The function of autophagy proteins in membrane formation and/ or trafficking is exploited by numerous viruses, including poliovirus, rotavirus, HIV, coronaviruses, Dengue virus, and the hepatitis B and C viruses $^{55}$. Autophagosomes (defined as LC3-positive membranes, see caveat below) may act as a scaffold for intracellular membrane-associated replication of certain cytoplasmic RNA viruses ${ }^{55}$. Autophagy may assist in HIV biogenesis, because the processing of the HIV envelope precursor protein Gag and extracellular viral release are enhanced by the autophagy machinery ${ }^{63}$. Similarly, autophagy proteins are required for maximal levels of poliovirus egress ${ }^{55}$. Another newly defined proviral function of autophagy is its role in productive hepatitis $C$ virus replication; several different autophagy proteins (such as beclin 1, ATG4B, ATG5, ATG7 and ATG12) assist in the translation of incoming, but not progeny, viral RNA ${ }^{64}$. ATG7 and class III PI(3)K activity also enhance hepatitis B virus DNA replication ${ }^{65}$.

The mechanisms by which autophagy proteins facilitate the replication and/or egress of certain viruses are not yet understood. Some observations may relate to the role of autophagy proteins in remodelling the ER (vis-à-vis viral replication) or the role of autophagosomes in fusing with multivesicular bodies (vis-à-vis viral egress). It is possible that autophagy proteins function to provide membrane for viral replication complexes or translation machinery. This may be true for viruses such as hepatitis $\mathrm{C}$ virus, for which genetic knockdown of several different autophagy genes decreases productive replication ${ }^{64}$. However, for other viruses such as coronaviruses, the biogenesis of double-membrane, ER-derived vesicles used for replication proceeds through a pathway that involves the nonlipidated form of LC3 (LC3-I) but not the general autophagy machinery ${ }^{66}$. Thus, caution must be exercised in interpreting the significance of the co-localization (or biochemical interaction) of viral proteins and LC3, as LC3 may have autophagy-independent roles in membrane dynamics.

\section{Autophagy regulation by immune signalling molecules}

The central importance of autophagy in immunity is further underscored by the multitude of immune-related signalling molecules that regulate autophagy. As reviewed in detail elsewhere ${ }^{2-4,38}$, autophagy is induced by different families of pathogen-recognition receptors (such as TLRs, NOD-like receptors and the double-stranded RNA-binding protein PKR), DAMPs (such as ATP, ROS and misfolded proteins), pathogen receptors (such as CD46), IFN- $\gamma$ and downstream immunityrelated GTPases, and DAP kinase, JNK, CD40, tumour necrosis factor- $\alpha$ (TNF- $\alpha$ ), inhibitor of NF- $\kappa B$ (IKK) and NF- $\kappa B$ (Fig. 1). High mobility group box (HMGB) proteins have also been shown to function as both universal sensors of nucleic acids in innate immune signalling ${ }^{67}$ and inducers of autophagy ${ }^{68}$. Autophagy is inhibited by BCL2, NF- $\kappa \mathrm{B}$, Thelper $2\left(\mathrm{~T}_{\mathrm{H}} 2\right)$ cytokines and the canonical nutrient-sensing insulinAKT-TOR pathway. Inactivation of this nutrient-sensing pathway may contribute to vesicular stomatitis virus stimulation of autophagy in Drosophila ${ }^{46}$, and autophagy activation in C. elegans with loss-of-function mutations in this pathway may mediate pathogen resistance in long-lived mutant nematodes ${ }^{48}$. Thus, both 'immune-specific' and more general nutrient-response signals control autophagy in response to infection.

Studies with vitamin D3 have uncovered a possible link between nutrition, innate immunity and the control of autophagy during mycobacterial infection. Low vitamin D3 levels are associated with increased susceptibility to tuberculosis. Vitamin D3 generates an antimycobacterial peptide, cathelicidin, and induces autophagy and mycobacterial killing in human monocytes through cathelicidin-dependent effects ${ }^{69}$. Although cathelicidin is required for vitamin-D3-dependent transcriptional upregulation of autophagy genes such as BECN1 and ATG5, and vitamin D3 enhances the recruitment of cathelicidin to autophagosomes, it is not yet clear how cathelicidin promotes autophagy. Nonetheless, these observations may begin to provide some insight into the century-old Nobel prize award (Niels Ryberg Finsen, 1903) for the use of ultraviolet-light therapy (which generates active vitamin D3) in the treatment of diseases such as tuberculosis.

In most instances, the mechanisms of autophagy control by immunerelated signalling molecules are not understood. However, there are some examples of specific interactions between immune signals and autophagy proteins that may be relevant to these mechanisms. For example, the interaction between beclin 1 and BCL2 (which inhibits its activity) is thought to be disrupted by the TLR adaptors MyD88 and TRIF, as well as by HMGB1, which bind to beclin 1 and displace BCL2 (refs 3, 68). Two intracellular sensors responsible for inducing autophagy in response to bacterial infection, NOD1 and NOD2, interact with ATG16L1 and recruit it to the plasma membrane, resulting in enhanced association of invasive bacteria (S. flexneri) with LC3 (ref. 53). Interestingly, a NOD2 mutation associated with Crohn's disease impairs ATG16L1 plasma membrane recruitment and bacterial co-localization with LC3 (ref. 53).

The identification of other possible protein-protein interactions between core autophagy proteins and immune signals by a large proteomics screen ${ }^{21}$ has the potential to foster further advances in understanding how different immune signals regulate the autophagy machinery. For example, tectonin proteins with multivalent 
$\beta$-propeller folds are known to function in pathogen recognition and innate immunity in invertebrates ${ }^{70}$. Thus, the interactions between previously uncharacterized human proteins of this tectonin family, TECPR1 and TECPR2, with the ATG5-ATG12-ATG16L1 complex and ATG8 family members, respectively ${ }^{21}$, may contribute to pathogen-induced autophagy stimulation or selective autophagic targeting of pathogens in mammals.

Further links between immune signalling molecules and autophagy regulation were suggested by a genome-wide short interfering RNA screen $^{71}$. The analysis identified 219 genes that suppressed basal autophagy, largely in a mammalian TOR complex 1 (mTORC1)independent fashion. These included several cytokines such as CLCF1, LIF, IGF1, FGF2 and the chemokine SDF1 (also known as CXCL12), as well as cellular signalling molecules regulated by cytokines such as STAT3. These findings raise the possibility that cytokines may have a broader role in the control of autophagy than previously thought. Moreover, because these cytokine signalling pathways are important in immune cells, another central question is to what extent cytokinemediated regulation of autophagy governs immune cell function. Given the general function of autophagy in cellular homeostasis ${ }^{5}$, and the more specific functions in regulating immune and inflammatory signalling (discussed in 'Regulation of immune signalling by autophagy proteins'), cytokine-mediated changes in autophagy levels in immune cells may have a central role in immunity and inflammation.

\section{Autophagy and adaptive immunity}

Autophagy proteins function in adaptive immunity, including in the development and homeostasis of the immune system and in antigen presentation (Table 1 and Fig. 3). The knockout of different autophagy genes in specific lymphocyte populations in mice has shown a crucial role for autophagy proteins in the maintenance of normal numbers of $\mathrm{B} 1 \mathrm{a} \mathrm{B}$ cells, $\mathrm{CD} 4^{+} \mathrm{T}$ cells, $\mathrm{CD} 8^{+} \mathrm{T}$ cells and fetal haematopoietic stem cells $^{2,44,72}$. In T cells, in which mitochondrial numbers are developmentally regulated during the transition from thymocyte to mature circulating T cell, the developmental defect in autophagy-deficient cells may be related to the defective clearance of mitochondria ${ }^{44}$. Another crucial function of autophagy in the development and homeostasis of the immune system is the elimination of autoreactive T cells in the thymus ${ }^{44}$. High levels of autophagy are present in thymic epithelial cells, in which autophagy participates in the delivery of self-antigens to MHC class II loading compartments. Genetic disruption of Atg5 in thymic epithelial cells leads to the altered selection of certain MHC class II restricted $\mathrm{T}$-cell specificities and autoimmunity ${ }^{73}$. Beyond these functions in lymphocyte survival and thymic negative selection, autophagy may exert other functions in lymphocyte differentiation, perhaps, in part, indirectly through effects on cytokine expression (see the next section). It is not yet known whether autophagy is involved in the development and/or homeostasis of immune cell populations other than lymphocytes and haematopoietic stem cells.

Autophagy proteins may participate in different facets of antigen presentation, including the delivery of endogenous antigens for MHC class II presentation to $\mathrm{CD} 4^{+} \mathrm{T}$ cells $\mathrm{s}^{74,75}$, the enhancement of antigen donor cell cross-presentation to $\mathrm{CD}^{+} \mathrm{T}$ cells ${ }^{75}$, dendritic cell crosspresentation of phagocytosed antigens to $\mathrm{CD} 4^{+} \mathrm{T}$ cells ${ }^{32}$ and, in one report, $\mathrm{MHC}$ class I presentation of intracellular antigens to $\mathrm{CD} 8^{+}$ $\mathrm{T}$ cells ${ }^{27}$. The discovery that autophagosomes could deliver endogenous antigens to MHC class II loading compartments sheds light on one of the central mysteries of antigen presentation - how the immune system elicits $\mathrm{CD} 4^{+} \mathrm{T}$-cell responses to antigens that originate in all parts of the cell. The autophagic delivery of endogenously synthesized antigens for MHC class II presentation has been demonstrated in vitro for certain viral antigens ${ }^{75}$, and probably explains the essential role of Atg5 in vivo in negative thymic selection ${ }^{73}$. However, the relative importance of this pathway in antigen presentation during infection in vivo is not yet known. There is nonetheless interest in exploiting this pathway for optimizing vaccine-elicited $\mathrm{CD} 4^{+} \mathrm{T}$-cell responses, by either pre- treating dendritic cells with autophagy-inducing agents in cell-based vaccine strategies or fusing antigens with LC3 to enhance their targeting to autophagosomes ${ }^{1}$.

Of note, autophagy proteins are required for antigen cross-presentation during infection in vivo ${ }^{32}$. Dendritic-cell-specific deletion of Atg5 in mice results in defects in priming $\mathrm{CD}^{+} \mathrm{T}$-cell responses after HSV and Listeria infections, and mice succumb more rapidly to lethal disease after intravaginal HSV infection. Atg5-deficient dendritic cells have normal migration, innate responses, endocytic and phagocytic activity and cross-presentation of peptides on MHC class I molecules. However, they exhibit defects in phagosome-to-lysosome fusion and in cross-presentation by MHC class II molecules of phagocytosed antigens containing TLR ligand. Thus, the interior of the phagosome, like that of the autophagosome, is a cellular compartment that autophagyprotein-dependent antigen presentation accesses to generate peptides for presentation to $\mathrm{CD} 4^{+} \mathrm{T}$ cells. A potential evolutionary advantage of this autophagy-protein-dependent cross-presentation is that, by delegating antigen presentation duties to uninfected dendritic cells, the host can bypass the blockade of antigen presentation that may result from microbial antagonism of autophagy in infected cells.

\section{Regulation of immune signalling by autophagy proteins}

In response to infection, the host must activate those arms of the innate and adaptive immune system (including autophagy-dependent functions; Fig. 3) that help to control infection while, in parallel, triggering specific responses that limit detrimental, uncontrolled immune activation and inflammation. An exciting new frontier in autophagy research is the growing recognition of the function of autophagy proteins in achieving this balance (Fig. 4).

Autophagy proteins function in both the activation and inactivation of innate immune signalling ${ }^{4}$. The autophagy pathway activates type I IFN production in plasmacytoid dendritic cells by delivering viral nucleic acids to endosomal TLRs ${ }^{76}$. By contrast, autophagy proteins negatively regulate RIG-I-like receptor (RLR)-mediated induction of type I IFN production through the autophagic elimination of damaged mitochondria (and reduction of ROS) ${ }^{77}$, and by the binding of ATG5ATG12 to caspase recruitment domains of RLR signalling molecules ${ }^{78}$. Moreover, the autophagy protein ATG9A, but not ATG7, negatively regulates the activation of STING, a transmembrane protein that is required for efficient activation of type I IFN and pro-inflammatory cytokine production in response to stimulatory $\mathrm{DNA}^{23}$. Thus, it seems that autophagy proteins can negatively regulate IFN production by both autophagy-dependent and -independent mechanisms. With respect to the latter, different autophagy proteins may be specialized to target different innate immune signalling molecules.

The autophagy pathway and/or proteins also have a crucial role in the control of inflammatory signalling. A major effect is on the regulation of inflammatory transcriptional responses. Increased levels of the adaptor protein $\mathrm{p} 62$, which accumulates in autophagy-deficient cells, activate the pro-inflammatory transcription factor NF- $\kappa \mathrm{B}$ through a mechanism involving TRAF6 oligomerization ${ }^{79}$. The accumulation of p62 in Atg7-deficient hepatocytes results in enhanced activity of the stress-responsive transcription factor NRF2 and NRF2-dependent liver injury ${ }^{80}$. In addition, Paneth cells (intestinal immune epithelial cells) from mice hypomorphic for Atg16l1 $\left(\operatorname{Atg} 1611^{\mathrm{HM}}\right)$ show enhanced transcription of pro-inflammatory cytokines and adipokines ${ }^{81}$.

A second important effect of autophagy proteins on inflammatory signalling is at the level of the inflammasome. This complex contains NOD-like receptor cryopyrin proteins, the adaptor protein ASC and caspase 1, and is activated by cellular infection or other stress to promote the maturation of pro-inflammatory cytokines such as interleukin-1 (IL-1 $\beta$ ) and IL-18 (ref. 4). Atg16l1- or Atg7deficient mouse macrophages produce increased levels of mature IL- $1 \beta$ and IL- 18 after TLR4 stimulation by endotoxin ${ }^{82}$. In addition, mouse chimaeras engrafted with $\operatorname{Atg} 16 l 1^{-/-}$fetal liver haematopoietic progenitors have increased serum concentrations of IL-1 $\beta$ and IL-18 


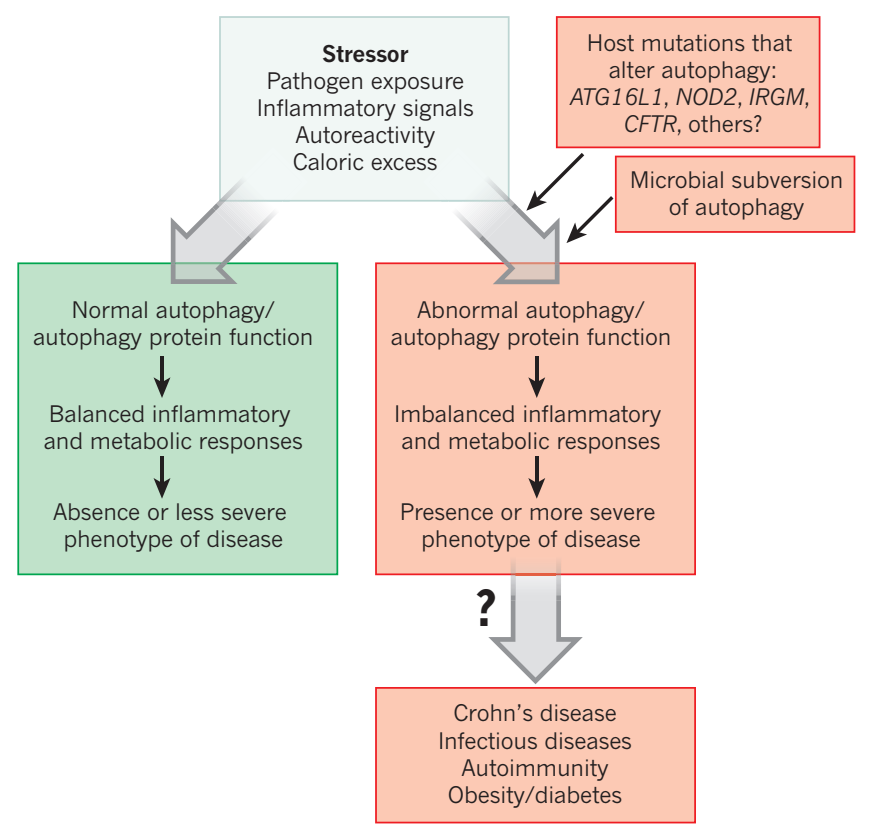

Figure 4 | Autophagy/autophagy proteins act to achieve a balance between activation and inactivation of innate immune signalling. A general model in which the levels of autophagy and autophagy proteins control disease in response to stressors. Normal autophagy protein function (green) contributes to balanced inflammatory and metabolic responses, resulting in protection against disease. Altered autophagy protein function (red) results in maladaptive inflammatory and metabolic responses, increased inflammation and more severe disease.

after treatment with dextran sodium sulphate (DSS), which contributes to increased DSS-induced colitis ${ }^{82}$.

The mechanism(s) by which autophagy proteins negatively regulate inflammasome activation are not yet understood. Mutually nonexclusive possibilities include direct interactions between autophagy proteins and inflammasome components, indirect inhibition of inflammasome activity through autophagic suppression of ROS accumulation, or autophagic degradation of danger signals that activate the inflammasome. In line with the latter model, the autophagic degradation of amyotrophic-lateral-sclerosis-linked mutant superoxide dismutase has been proposed to limit caspase 1 activation and IL- $1 \beta$ production ${ }^{83}$.

In addition to regulating inflammatory signalling, the autophagy pathway may prevent tissue inflammation through its role in apoptotic corpse clearance. The efficient clearance of apoptotic corpses during development and tissue homeostasis prevents secondary necrosis, which releases danger signals (DAMPs) that trigger inflammation. Autophagy genes are essential for the heterophagic clearance of dying apoptotic cells during developmental programmed cell death (by the generation of ATP-dependent engulfment signals) ${ }^{84}$, and the retinas and lungs of embryonic mice lacking Atg5 have a defect in apoptotic corpse engulfment that is associated with infiltration of inflammatory cells ${ }^{84}$. On the basis of growing evidence that autophagy proteins function in TLRmediated phagolysosomal pathways, it is possible that autophagy also functions in phagocytes to facilitate apoptotic corpse clearance. Thus, in tissues such as the intestine, in which physiological regeneration involves continuous shedding or apoptosis of epithelial cells, autophagydependent functions in dying cells and/or phagocytic cells may promote efficient corpse clearance, thereby limiting inflammation.

\section{Autophagy and inflammatory disease}

Perturbations in autophagy-protein-dependent functions in immunity may contribute not only to increased susceptibility to infection, but also to chronic inflammatory diseases and autoimmune diseases. The only well-characterized link thus far is between mutations in autophagy regulators and Crohn's disease, a chronic inflammatory disorder of the small intestine, in which a breakdown in clearance or recognition of commensal bacteria, as well as altered mucosal barrier function and cytokine production, is thought to lead to intestinal inflammation (Fig. 5). Other emerging links include the autoimmune disease systemic lupus erythematosus (SLE), inflammation-associated metabolic diseases such as obesity and diabetes, and inflammation associated with cystic fibrosis lung disease (Fig. 4).

The role of autophagy proteins in Crohn's disease was not suspected until genome-wide association studies identified three Crohn's disease susceptibility genes, IRGM, NOD2 and ATG16L1, that are involved in autophagy ${ }^{85}$. The IRGM risk allele contains a deletion in the promoter region of the gene that may be associated with changes in IRGM protein expression and may contribute to Crohn's disease, given IRGM's role in autophagy-dependent control of bacterial infection ${ }^{49}$. However, this hypothesis has not yet been tested. The three major Crohn's-diseaseassociated NOD2 variants (a frameshift mutant and two missense mutants) may be loss-of-function mutants, with impaired muramyl dipeptide (MDP)-induced inflammatory signalling ${ }^{86}$. How the loss of function of a pro-inflammatory signal mechanistically contributes to an inflammatory disorder has been unclear, but the recently discovered links between NOD2 and autophagy may solve this conundrum. In primary immature human dendritic cells, NOD2 is required for MDPinduced autophagy, a process that is essential for the MHC class II presentation of bacterial antigens to $\mathrm{CD} 4^{+} \mathrm{T}$ cells and for bacterial targeting to lysosomes ${ }^{52}$. Dendritic cells expressing Crohn's disease NOD2 risk variants are defective in both of these functions ${ }^{52}$. Thus, in patients with Crohn's disease and NOD2 risk variants, aberrant autophagy-dependent bacterial clearance and immune priming could act as a trigger for intestinal inflammation.

A mechanistic link may also exist between ATG16L1 mutation and Crohn's disease pathogenesis. Similar to findings with NOD2 variants, dendritic cells from patients with the Crohn's-disease-associated ATG16L1(T300A) risk variant are defective in presenting bacterial antigen to $\mathrm{CD} 4^{+} \mathrm{T}$ cells ${ }^{52}$. However, it is not yet known how the T300A mutation affects the function of the mammalian ATG16L1 protein. This mutation resides in the carboxy-terminal WD-repeat domain that is absent in yeast Atg16 and is dispensable for autophagy. Although some studies have suggested that the $A T G 16 L 1(T 300 A)$ variant has reduced autophagic clearance of enteric pathogens such as adherent-invasive Escherichia coli $^{87}$ or $S$. Typhimurium ${ }^{88}$, it remains controversial whether the risk versus protective alleles of $A T G 16 L 1$ have differences in stability or antibacterial autophagic activity ${ }^{89}$.

Despite the uncertain nature of the effects of the T300A mutation on ATG16L1 function, Atg16l1 mutation (null or hypomorphic alleles) in mice results in abnormalities that are relevant to Crohn's disease pathogenesis. As noted earlier, loss of Atg1611 function in mice results in enhanced TLR-agonist-induced pro-inflammatory cytokine production by macrophages ${ }^{82}$, enhanced DSS-induced colitis ${ }^{82,90}$ and altered inflammatory gene transcriptional profiles in Paneth cells ${ }^{81,90}$. In addition, the Paneth cells of mice expressing low Atg16l1 levels $\left(\operatorname{Atg} 16 l 1^{\mathrm{HM}}\right.$ ) show defects in the packaging and extrusion of antimicrobial granules into the gut lumen; Paneth cells from patients with Crohn's disease and the ATG16L1(T300A) risk variant show similar defects ${ }^{81}$. This suggests that, in addition to the overlapping functions of NOD2 and ATG16L1 in a common bacterial-sensing pathway that promotes bacterial antigen presentation, ATG16L1 may have unique protective functions, including Paneth cell antimicrobial peptide release and the negative regulation of pro-inflammatory cytokine production. To connect the striking phenotypes in Atg1611-mutant mice and the pathogenesis of Crohn's disease in humans with the ATG16L1(T300A) risk allele, the precise effects of the T300A mutation on ATG16L1 protein function need to be uncovered.

A new dimension in understanding the multifactorial basis of chronic inflammatory diseases such as Crohn's disease has emerged from the discovery that a virus trigger is required to observe intestinal 


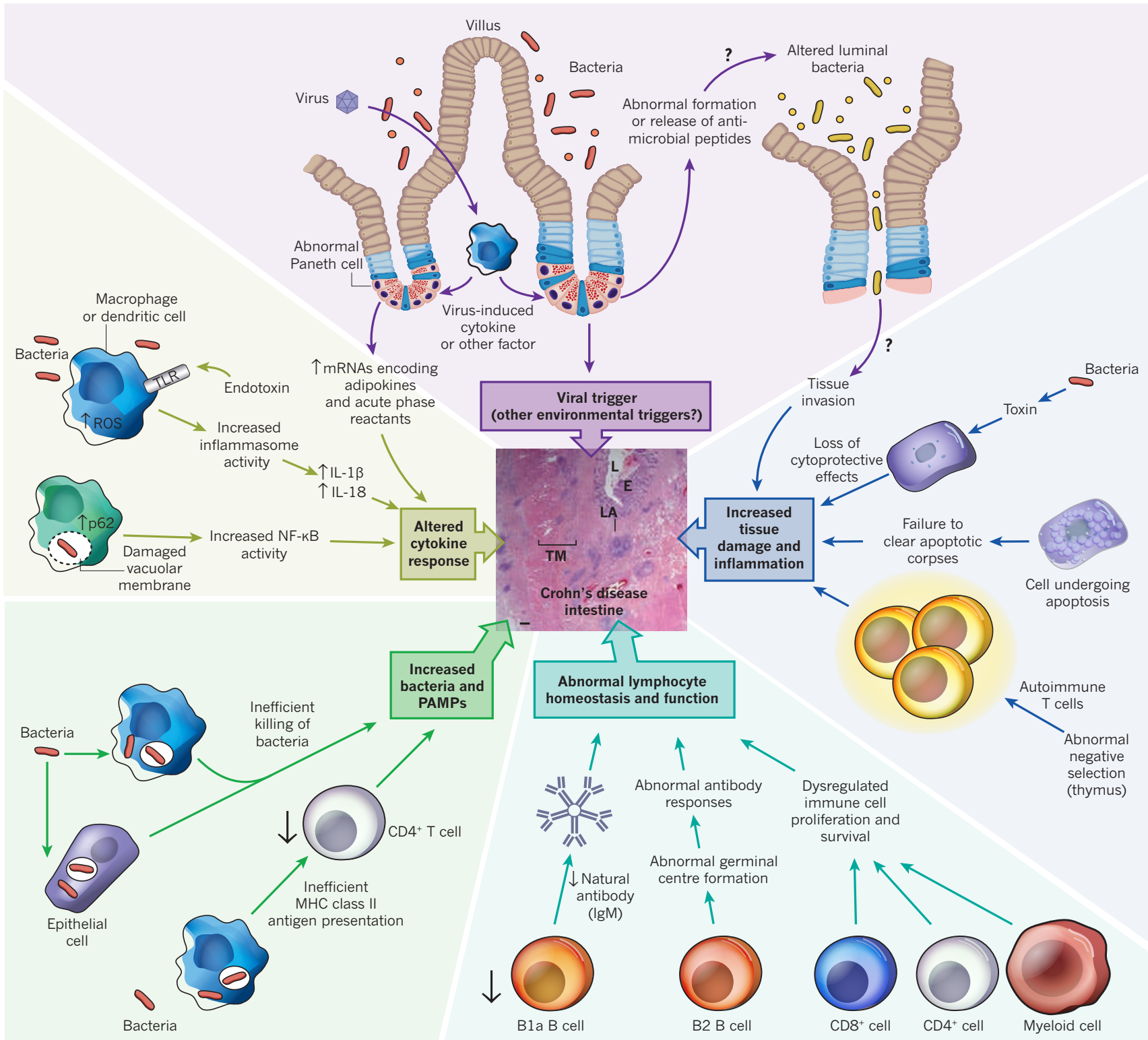

Figure 5 | The link between mutations in autophagy regulators and the chronic inflammatory disorder Crohn's disease. An overview of the many possible mechanisms by which defects in autophagy and autophagy protein function may contribute to the pathogenesis of a type of inflammatory bowel disease, Crohn's disease. A micrograph of a human small intestine from a patient with Crohn's disease is shown (centre), demonstrating the severe transmural inflammation that is characteristic of this disease. The postulated

abnormalities in $\operatorname{Atg} 16 l 1^{\mathrm{HM}}$ mice ${ }^{90}$. In mice raised in a pathogen-free facility, only $\operatorname{Atg} 16 l 1^{\mathrm{HM}}$ mice (and not wild-type mice) infected with a virus found in routine conventional animal facilities, a murine norovirus, showed abnormal Paneth cell granule secretion, Paneth cell proinflammatory gene-expression profiles, and intestinal inflammation in response to DSS treatment ${ }^{90}$. This mucosal inflammation depended on the presence of the microbiome and pro-inflammatory cytokines, as it was reversed by antibiotic treatment or by TNF- $\alpha$ or IFN- $\gamma$ inhibition. Thus, variations in a host autophagy gene, exposure to a specific virus and the microbiome can act together to trigger intestinal inflammation in mice that is similar to that in patients with Crohn's disease. Although environmental factors, including the gut microbiome, have long been suspected to contribute to Crohn's disease in genetically susceptible individuals, formal proof of this concept was lacking, and viruses were a previously unsuspected trigger. Another implication of this work is the mechanisms by which defects in autophagy protein function might contribute to the development or perpetuation of intestinal inflammation are based on studies in vitro and animal models. There is no direct evidence that autophagy defects contribute to human Crohn's disease, although mutations in three autophagy-related genes, ATG16L1, NOD2 and IRGM, are known to enhance risk of the disease. E, epithelium; IgM, immunoglobulin M; L, lumen; LA, lymphoid aggregates; TM, thickened muscle. Scale bar, $200 \mu \mathrm{m}$.

concept that autophagy proteins, through their diverse roles in immunity and the control of inflammation, may serve as a central rheostat that prevents inflammatory diseases triggered by environmental stress (Fig. 4).

An important unanswered question is whether perturbations in autophagy may also result in inflammatory autoimmune disease. Genome-wide association studies have linked several single nucleotide polymorphisms (SNPs) in ATG5 to SLE susceptibility ${ }^{91-93}$. SLE is a multifactorial, heterogeneous disease characterized by autoimmune responses against self-antigens generated from dying cells. Although the effects of these SNPs on ATG5 expression and function are not known, the lack of Atg5-dependent negative thymic selection generates autoimmunity and multi-organ inflammation in mice ${ }^{73}$. Loss of other ATG5dependent effects, including regulation of IFN and pro-inflammatory cytokine secretion $^{77,78}$, clearance of dying cells ${ }^{84}$ and dendritic cell 
antigen presentation ${ }^{32}$, might also contribute to the autoimmunity and inflammation associated with SLE. Thus, a link between ATG5 mutation (or mutation of other autophagy genes) and SLE pathogenesis is biologically plausible, although not yet proven.

Defects in autophagy may contribute to inflammation-associated metabolic diseases such as diabetes and obesity, which are both linked to insulin resistance. The metabolic inflammasome - a complex composed of signalling molecules such as PKR, eIF2 $\alpha$, JNK, IRS and IKKmay act as a link between ER stress and more global stress responses, including inflammation and metabolic dysfunction (as observed in insulin resistance and obesity $)^{94}$. Although most components of the metabolic inflammasome promote autophagy, the induction of autophagy by this signalling complex would be expected to serve as a negativefeedback mechanism that limits ER stress and disease progression. Consistent with this postulated protective effect of autophagy, hepatic suppression of the autophagy gene Atg7 in mice results in increased ER stress and insulin resistance ${ }^{95}$, and mice deficient in the autophagy adaptor protein p62 develop mature-onset obesity and insulin resistance ${ }^{96}$. Furthermore, obesity is associated with the accumulation and activation of macrophages and subsets of T cells in adipose tissue and the production of cytokines such as TNF- $\alpha$ and IL-6 (ref. 97). Thus, the failure of autophagy-dependent control of ER stress, immune cell homeostasis, immune cell activation and/or pro-inflammatory cytokine secretion may contribute to inflammation-associated responses that underlie the pathogenesis of metabolic diseases.

Another potential link between autophagy deficiency and chronic inflammation is in cystic fibrosis ${ }^{98}$, a life-threatening genetic disorder caused by mutations in the gene encoding the cystic fibrosis transmembrane conductance regulator (CFTR). Mutations in CFTR lead to autophagy inhibition in lung epithelial cells through a mechanism that may involve ROS-mediated sequestration of the beclin 1-class III $\mathrm{PI}(3) \mathrm{K}$ complex in perinuclear aggregates (redirecting it from its site of autophagy action at the ER). Restoration of beclin 1 and autophagy in cystic fibrosis epithelial cells rescues the disease phenotype, and antioxidants reverse the airway inflammation in a cystic fibrosis mouse model by a mechanism postulated to involve autophagy.

\section{Future directions}

The first series of studies demonstrating that the autophagy machinery is used to attack invading intracellular bacteria was published in 2004 (refs 30, 99, 100). Although autophagy had been observed at the ultrastructural level in cells infected with intracellular bacteria and viruses decades earlier, these studies were a seminal advance. For the first time, pharmacological and genetic manipulation of autophagy, which built on the discoveries of the yeast screens that identified the autophagy machinery, challenged the very notion of autophagy as an 'auto'- (self), 'phagy' (eating) pathway. Indeed, we learned that the same genes that are used to orchestrate the degradation of self-constituents, either for nutritional/energy homeostasis or cellular damage control, are also used to orchestrate the degradation of foreign invaders, termed xenophagy.

In the past few years, research in the field has uncovered new layers of complexity and functional diversity in terms of how this set of genes originally characterized in the context of macroautophagy - may function to protect multicellular organisms against not only the threats of infection but also the threats of the host's own response to infection. The autophagy machinery does much more than form autophagosomes to engulf microbes - it somehow allows microbes in phagosomes and vacuoles to be targeted to the lysosome; it enables crucial cells in the immune system to develop properly and perform some of their 'normal' functions (such as produce IFN, secrete antimicrobial peptides or present antigens to stimulate adaptive immunity); and it ensures that these responses do not become out of control by functioning in central immunological tolerance and the negative regulation of innate and inflammatory signalling. Thus, recent advances may not only modify our understanding of immunity (in terms of understanding new roles of the autophagy machinery in immune regulation) but also reshape our understanding of the pathogenesis of inflammatory diseases (in terms of understanding how perturbations in autophagy protein function may contribute to such diseases).

Clearly, our understanding of the molecular mechanisms of the plethora of functions of autophagy proteins in immune-related processes is still quite primitive. We speculate that, similar to the way in which the initial genetic screens in yeast transformed autophagy research, current proteomic and genomic screens have the potential to transform research on autophagy and immunity. Such a transformation would include facilitating a much deeper understanding of the molecular mechanisms of the existing known immunological functions of autophagy through the use of the tools of modern systems biology to understand autophagy protein-protein interaction and signalling regulatory networks on a broad scale. Perhaps more exciting is the possibility that such a transformation will uncover new ways in which this ancient self-defence machinery can function in immunity.

1. Deretic, V. \& Levine, B. Autophagy, immunity, and microbial adaptations. Cell Host Microbe 5, 527-549 (2009).

2. Virgin, H. W. \& Levine, B. Autophagy genes in immunity. Nature Immunol. 10, 461-470 (2009).

3. Kroemer, G., Marino, G. \& Levine, B. Autophagy and the integrated stress response. Mol. Cell 40, 280-293 (2010).

4. Saitoh, T. \& Akira, S. Regulation of innate immune responses by autophagyrelated proteins. J. Cell Biol. 189, 925-935 (2010).

5. Levine, B. \& Kroemer, G. Autophagy in the pathogenesis of disease. Cell $\mathbf{1 3 2}$ 27-42 (2008).

6. Mizushima, N., Yoshimori, T. \& Levine, B. Methods in mammalian autophagy research. Cell 140, 313-326 (2010).

This paper provides a concise and critical review of current methods to monitor and modulate autophagy in mammalian cells.

7. Schmid, D., Pypaert, M. \& Munz, C. Antigen-loading compartments for major histocompatibility complex class II molecules continuously receive input from autophagosomes. Immunity 26, 79-92 (2007).

8. Yu, L. et al. Termination of autophagy and reformation of lysosomes regulated by mTOR. Nature 465, 942-946 (2010).

9. Nakatogawa, H., Suzuki, K., Kamada, Y. \& Ohsumi, Y. Dynamics and diversity in autophagy mechanisms: lessons from yeast. Nature Rev. Mol. Cell Biol. 10, 458-467 (2009).

10. Hayashi-Nishino, M. et al. A subdomain of the endoplasmic reticulum forms a cradle for autophagosome formation. Nature Cell Biol. 11, 1433-1437 (2009).

11. Yla-Antilla, P., Vihinen, H., Jokitalo, E. \& Eskelinen, E. L. 3D tomography reveals connections between the phagophore and endoplasmic reticulum. Autophagy 5, 1180-1185 (2009)

12. Mizushima, N. The role of the Atg1/ULK1 complex in autophagy regulation. Curr. Opin. Cell Biol. 22, 132-139 (2010)

13. Itakura, E. \& Mizushima, N. Characterization of autophagosome formation site by a hierarchical analysis of mammalian Atg proteins. Autophagy 6, 764-776 (2010).

14. Matsunaga, K. et al. Two Beclin 1-binding proteins, Atg14L and Rubicon, reciprocally regulate autophagy at different stages. Nature Cell Biol. 11, 385-396 (2009)

15. Axe, E. L. et al. Autophagosome formation from membrane compartments enriched in phosphatidylinositol 3-phosphate and dynamically connected to the endoplasmic reticulum. J. Cell Biol. 182, 685-701 (2008).

16. Polson, H. E. et al. Mammalian Atg18 (WIPI2) localizes to omegasome-anchored phagophores and positively regulates LC3 lipidation. Autophagy 6, 506-522 (2010).

17. Ropolo, A. et al. The pancreatitis-induced vacuole membrane protein 1 triggers autophagy in mammalian cells. J. Biol. Chem. 282, 124-133 (2007).

18. Tian, Y. et al. C. elegans screen identifies autophagy genes specific to multicellular organisms. Cell 141, 1042-1055 (2010).

19. Fujita, N. et al. The Atg16L complex specifies the site of LC3 lipidation for membrane biogenesis in autophagy. Mol. Biol. Cell 19, 2092-2100 (2008).

20. Weidberg, H. et al. LC3 and GATE-16/GABARAP subfamilies are both essential yet act differently in autophagosome biogenesis. EMBO J. 29, 1792-1802 (2010).

21. Behrends, C., Sowa, M. E., Gygi, S. P. \& Harper, J. W. Network organization of the human autophagy system. Nature 466, 68-76 (2010).

22. Radoshevich, L. et al. ATG12 conjugation to ATG3 regulates mitochondrial homeostasis and cell death. Cell 142, 590-600 (2010).

23. Saitoh, T. et al. Atg9a controls dsDNA-driven dynamic translocation of STING and the innate immune response. Proc. Natl Acad. Sci. USA 106, 20842-20846 (2009).

24. Webber, J. L. \& Tooze, S. A. New insights into the function of Atg9. FEBS Lett 584, 1319-1326 (2010).

25. Hailey, D. W. et al. Mitochondria supply membranes for autophagosome biogenesis during starvation. Cell 141, 656-667 (2010)

26. Ravikumar, B., Moreau, K., Jahreiss, L., Puri, C. \& Rubinsztein, D. C. Plasma membrane contributes to the formation of pre-autophagosomal structures. Nature Cell Biol. 12, 747-757 (2010).

27. English, L. et al. Autophagy enhances the presentation of endogenous viral 
antigens on MHC class I molecules during HSV-1 infection. Nature Immunol. 10, 480-487 (2009).

28. Kraft, C., Peter, M. \& Hofmann, K. Selective autophagy: ubiquitin-mediated recognition and beyond. Nature Cell Biol. 12, 836-841 (2010).

29. Yamaguchi, H. et al. An initial step of GAS-containing autophagosome-like vacuoles formation requires Rab7. PLoS Pathogens 5, e1000670 (2009).

30. Nakagawa, I. et al. Autophagy defends cells against invading group A Streptococcus. Science 306, 1037-1040 (2004)

Reference 30, together with reference 99, provides the first evidence that autophagy has a key role in bacterial infection. Atg5 is shown to be essentia for controlling the replication of group A Streptococci that escape into the cytoplasm.

31. Sanjuan, M. A. et al. Toll-like receptor signalling in macrophages links the autophagy pathway to phagocytosis. Nature 450, 1253-1257 (2007).

32. Lee, H. K. et al. In vivo requirement for Atg 5 in antigen presentation by dendritic cells. Immunity 32, 227-239 (2010).

This study shows that the autophagy machinery is necessary for dendritic cells to process and present extracellular microbial antigens for MHC class II presentation in vivo, which protects mice against lethal viral infection.

33. Berger, S. B. et al. SLAM is a microbial sensor that regulates bacterial phagosome functions in macrophages. Nature Immunol. 11, 920-927 (2010).

34. Huang, J. et al. Activation of antibacterial autophagy by NADPH oxidases. Proc. Natl Acad. Sci. USA 106, 6226-6231 (2009).

35. Zhao, Z. et al. Autophagosome-independent essential function for the autophagy protein Atg5 in cellular immunity to intracellular pathogens. Cell Host Microbe 4, 458-469 (2008).

36. Khaminets, A. et al. Coordinated loading of IRG resistance GTPases on to the Toxoplasma gondii parasitophorous vacuole. Cell. Microbiol. 12, 939-961 (2010)

37. Zhao, Y. O., Khaminets, A., Hunn, J. P. \& Howard, J. C. Disruption of the Toxoplasma gondii parasitophorous vacuole by IFNy-inducible immunity-related GTPases (IRG proteins) triggers necrotic cell death. PLoS Pathogens 5, 1-17 (2009).

38. Sumpter, R. Jr \& Levine, B. Autophagy and innate immunity: triggering targeting and tuning. Semin. Cell Dev. Biol. 21, 699-711 (2010).

39. Shahnazari, S. et al. A diacylglycerol-dependent signaling pathway contributes to regulation of antibacterial autophagy. Cell Host Microbe 8, 137-146 (2010).

40. Orvedahl, A. et al. Autophagy protects against Sindbis virus infection of the central nervous system. Cell Host Microbe 7, 115-127 (2010).

41. Dupont, N. et al. Shigella phagocytic vacuolar membrane remnants participate in the cellular response to pathogen invasion and are regulated by autophagy. Cell Host Microbe 6, 137-149 (2009).

42. Ponpuak, M. et al. Delivery of cytosolic components by autophagic adaptor protein p62 endows autophagosomes with unique antimicrobial properties. Immunity 32, 329-341 (2010).

43. Guillemain, A. \& Plus, N. Contrôle génique de la multiplication du virus de la sensibilité héréditaire au $\mathrm{CO}_{2}$ chez Drosophila melanogaster. Caryologia (suppl.) 1211-1213 (1954)

44. Mizushima, N. \& Levine, B. Autophagy in mammalian development and differentiation. Nature Cell Biol. 12, 823-830 (2010)

45. Liu, Y. et al. Autophagy regulates programmed cell death during the plant innate immune response. Cell 121, 567-577 (2005).

46. Shelly, S., Lukinova, N., Bambina, S., Berman, A. \& Cherry, S. Autophagy is an essential component of Drosophila immunity against vesicular stomatitis virus. Immunity 30, 588-598 (2009).

47. Yano, T. et al. Autophagic control of listeria through intracellular innate immune recognition in drosophila. Nature Immunol. 9, 908-916 (2008).

48. Jia, K. et al. Autophagy genes protect against Salmonella typhimurium infection and mediate insulin signaling-regulated pathogen resistance. Proc. Natl Acad. Sci. USA 106, 14564-14569 (2009).

49. Singh, S. B., Davis, A. S., Taylor, G. A. \& Deretic, V. Human IRGM induces autophagy to eliminate intracellular mycobacteria. Science 313, 1438-1441 (2006).

50. Intemann, C. D. et al. Autophagy gene variant IRGM -261T contributes to protection from tuberculosis caused by Mycobacterium tuberculosis but not by M. africanum strains. PLoS Pathogens 5, e1000577 (2009).

51. Kumar, D. et al. Genome-wide analysis of the host intracellular network that regulates survival of Mycobacterium tuberculosis. Cell 140, 731-743 (2010).

52. Cooney, R. et al. NOD2 stimulation induces autophagy in dendritic cells influencing bacterial handling and antigen presentation. Nature Med. 16, 90-97 (2010)

53. Travassos, L. H. et al. Nod1 and Nod2 direct autophagy by recruiting ATG16L1 to the plasma membrane at the site of bacterial entry. Nature Immunol. 11, 55-62 (2010).

54. Zhang, F. R. et al. Genomewide association study of leprosy. N. Engl. J. Med. 361 , 2609-2618 (2009)

55. Dreux, M. \& Chisari, F. V. Viruses and the autophagy machinery. Cell Cycle $\mathbf{9}$, 1295-1307 (2010)

56. Blanchet, F. P. etal. Human immunodeficiency virus-1 inhibition of immunoamphisomes in dendritic cells impairs early innate and adaptive immune responses. Immunity 32, 654-669 (2010)

57. Orvedahl, A. et al. HSV-1 ICP34.5 confers neurovirulence by targeting the Beclin 1 autophagy protein. Cell Host Microbe 1, 23-35 (2007).

58. Leib, D. A., Alexander, D. E., Cox, D., Yin, J. \& Ferguson, T. A. Interaction of ICP34.5 with Beclin 1 modulates herpes simplex virus type 1 pathogenesis through control of $\mathrm{CD4}^{+} \mathrm{T}$ cell responses. J. Virol. 83, 12164-12171 (2009).
59. E. X. et al. Viral Bcl-2-mediated evasion of autophagy aids chronic infection of hherpesvirus 68. PLoS Pathogens 5, e1000609 (2009)

60. Lee, J. S. et al. FLIP-mediated autophagy regulation in cell death control. Nature Cell Biol. 11, 1355-1362 (2009).

61. Yoshikawa, Y. et al. Listeria monocytogenes ActA-mediated escape from autophagic recognition. Nature Cell Biol. 11, 1233-1240 (2009).

62. Moreau, K. et al. Autophagosomes can support Yersinia pseudotuberculosis replication in macrophages. Cell. Microbiol. 12, 1108-1123 (2010).

63. Kyei, G. B. et al. Autophagy pathway intersects with HIV-1 biosynthesis and regulates viral yields in macrophages. J. Cell Biol. 186, 255-268 (2009).

64. Dreux, M., Gastaminza, P., Wieland, S. F. \& Chisari, F. V. The autophagy machinery is required to initiate hepatitis $\mathrm{C}$ virus replication. Proc. Natl Acad. Sci. USA 106, 14046-14051 (2009).

This study shows that many autophagy proteins are essential for the initial stages of hepatitis C viral RNA translation, illustrating that autophagy proteins may be subverted to enhance the replication of intracellular pathogens.

65. Sir, D. et al. The early autophagic pathway is activated by hepatitis B virus and required for viral DNA replication. Proc. Natl Acad. Sci. USA 107, 4383-4388 (2010).

66. Reggiori, F. et al. Coronaviruses hijack the LC3-I-positive EDEMosomes, ER-derived vesicles exporting short-lived ERAD regulators, for replication. Cell Host Microbe 7, 500-508 (2010).

67. Yanai, H. et al. HMGB proteins function as universal sentinels for nucleic-acidmediated innate immune responses. Nature 462, 99-103 (2009).

68. Tang, D. et al. Endogenous HMGB1 regulates autophagy. J. Cell Biol. 190, 881-892 (2010)

69. Yuk, J. M. et al. Vitamin D3 induces autophagy in human monocytes/ macrophages via cathelicidin. Cell Host Microbe 6, 231-243 (2009).

70. Low, D. H. et al. A novel human tectonin protein with multivalent $\beta$-propeller folds interacts with ficolin and binds bacterial LPS. PLOS ONE 4, e6260 (2009).

71. Lipinski, M. M. et al. A genome-wide siRNA screen reveals multiple mTORC1 independent signaling pathways regulating autophagy under normal nutritional conditions. Dev. Cell 18, 1041-1052 (2010).

72. Liu, F. et al. FIP200 is required for the cell-autonomous maintenance of fetal hematopoietic stem cells. Blood 116, 4806-4814 (2010).

73. Nedjic, J., Aichinger, M., Emmerich, J., Mizushima, N. \& Klein, L. Autophagy in thymic epithelium shapes the T-cell repertoire and is essential for tolerance. Nature 455, 396-400 (2008)

This study provided the first evidence that the autophagy machinery function in MHC class II antigen presentation in vivo, specifically in shaping the CD4 T-cell repertoire during negative thymic selection, and thereby preventing autoimmunity and multi-organ inflammation.

74. Paludan, C. et al. Endogenous MHC class II processing of a viral nuclear antigen after autophagy. Science 307, 593-596 (2005).

This study provided the first evidence that the autophagy machinery can deliver endogenously synthesized antigens for presentation on MHC class II molecules to $\mathrm{CD} 4^{+} \mathrm{T}$ cells.

75. Munz, C. Antigen processing via autophagy-not only for MHC class II presentation anymore? Curr. Opin. Immunol. 22, 89-93 (2010).

76. Lee, H. K., Lund, J. M., Ramanathan, B., Mizushima, N. \& Iwasaki, A. Autophagydependent viral recognition by plasmacytoid dendritic cells. Science $\mathbf{3 1 5}$, 1398-1401 (2007)

77. Tal, M. C. et al. Absence of autophagy results in reactive oxygen speciesdependent amplification of RLR signaling. Proc. Natl Acad. Sci. USA 106, 2770-2775 (2009)

78. Jounai, N. et al. The Atg5-Atg12 conjugate associates with innate antiviral immune responses. Proc. Natl Acad. Sci. USA 104, 14050-14055 (2007)

79. Moscat, J. \& Diaz-Meco, M. T. p62 at the crossroads of autophagy, apoptosis, and cancer. Cell 137, 1001-1004 (2009).

80. Komatsu, M. et al. The selective autophagy substrate $p 62$ activates the stress responsive transcription factor Nrf2 through inactivation of Keap1. Nature Cell Biol. 12, 213-223 (2010)

81. Cadwell, K. et al. A key role for autophagy and the autophagy gene Atg16/1 in mouse and human intestinal Paneth cells. Nature 456, 259-263 (2008).

82. Saitoh, T. et al. Loss of the autophagy protein Atg16L1 enhances endotoxininduced IL-1 $\beta$ production. Nature 456, 264-268 (2008).

This study demonstrates that autophagy proteins negatively control endotoxin-induced inflammasome activation. References 81 and 82 also show that autophagy gene deficiency increases susceptibility to experimentally induced inflammatory bowel disease.

83. Meissner, F., Molawi, K. \& Zychlinsky, A. Mutant superoxide dismutase 1 induced IL-1 $\beta$ accelerates ALS pathogenesis. Proc. Natl Acad. Sci. USA 107, 13046-13050 (2010).

84. Qu, X. et al. Autophagy gene-dependent clearance of apoptotic cells during embryonic development. Cell 128, 931-946 (2007).

This paper shows that autophagy genes are necessary for apoptotic cells to generate engulfment signals required for successful apoptotic corpse clearance and the prevention of tissue inflammation.

85. Barrett, J. C. et al. Genome-wide association defines more than 30 distinct susceptibility loci for Crohn's disease. Nature Genet. 40, 955-962 (2008).

86. Brain, O., Allan, P. \& Simmons, A. NOD2-mediated autophagy and Crohn disease. Autophagy 6, 412-414 (2010).

87. Lapaquette, P., Glasser, A. L., Huett, A., Xavier, R. J. \& Darfeuille-Michaud, A. Crohn's disease-associated adherent-invasive $E$. coli are selectively favoured by impaired autophagy to replicate intracellularly. Cell. Microbiol. 12, 99-113 (2010). 
88. Kuballa, P., Huett, A., Rioux, J. D., Daly, M. J. \& Xavier, R. J. Impaired autophagy of an intracellular pathogen induced by a Crohn's disease associated ATG16L1 variant. PLOS ONE 3, e3391 (2008).

89. Fujita, N. et al. Differential involvement of Atg16L1 in Crohn disease and canonical autophagy: analysis of the organization of the Atg16L1 complex in fibroblasts. J. Biol. Chem. 284, 32602-32609 (2009).

90. Cadwell, K. et al. Virus-plus-susceptibility gene interaction determines Crohn's disease gene Atg16L1 phenotypes in intestine. Cell 141, 1135-1145 (2010). This study shows that both hypomorphic expression of an autophagy protein and a viral-infection trigger are necessary for experimentally induced inflammatory bowel disease, suggesting that the interaction between host defects in autophagy and environmental stressors such as infection may be crucial for the pathogenesis of certain inflammatory diseases.

91. Harley, J. B. et al. Genome-wide association scan in women with systemic lupus erythematosus identifies susceptibility variants in ITGAM, PXK, KIAA1542 and other loci. Nature Genet. 40, 204-210 (2008).

92. Gateva, V. et al. A large-scale replication study identifies TNIP1, PRDM1, JAZF1, UHRFIBPI and IL 10 as risk loci for systemic lupus erythematosus. Nature Genet. 41, 1228-1233 (2009).

93. Han, J. W. et al. Genome-wide association study in a Chinese Han population identifies nine new susceptibility loci for systemic lupus erythematosus. Nature Genet. 41, 1234-1237 (2009).

94. Nakamura, T. et al. Double-stranded RNA-dependent protein kinase links pathogen sensing with stress and metabolic homeostasis. Cell 140, 338-348 (2010).

95. Yang, L., Li, P., Fu, S., Calay, E. S. \& Hotamisligil, G. S. Defective hepatic autophagy in obesity promotes ER stress and causes insulin resistance. Cell Metab. 11, 467-478 (2010).

96. Rodriguez, A. et al. Mature-onset obesity and insulin resistance in mice deficient in the signaling adapter p62. Cell Metab. 3, 211-222 (2006).

97. Hotamisligil, G. S. Inflammation and metabolic disorders. Nature 444, 860-867 (2006).

98. Luciani, A. et al. Defective CFTR induces aggresome formation and lung inflammation in cystic fibrosis through ROS-mediated autophagy inhibition. Nature Cell Biol. 12, 863-875 (2010).

99. Gutierrez, M. G. et al. Autophagy is a defense mechanism inhibiting BCG and Mycobacterium tuberculosis survival in infected macrophages. Cell 119, 753-766 (2004).

Reference 99, together with reference 30 , provides the first evidence that autophagy has a key role in bacterial infection. Autophagy induction by IFN-y, rapamycin or starvation results in the conversion of mycobacterial phagosomes into phagolysosomes, thereby enhancing mycobacterial killing.

100. Ogawa, M. et al. Escape of intracellular Shigella from autophagy. Science 307, 727-731 (2005).

Acknowledgements The work in the authors' laboratories was supported by National Institutes of Health (NIH) grants RO1 CA109618 and U54 AI057156 (B.L.); by Grants-in-Aid for Scientific Research from the Ministry of Education, Culture, Sports, Science and Technology, Japan, and by the Takeda Science Foundation (N.M.); and by NIH grants R01 AI054483, U54 AI057160, RO1 AI084887 and RO1 CA096511 and the Broad Medical Foundation (H.W.V.). We thank T. Stappenbeck for discussions and A. Diehl and M. Harstein for scientific illustration. We apologize to those authors whose work could not be cited owing to space limitations.

Author Information Reprints and permissions information is available at www.nature.com/reprints. The authors declare no competing interests. Correspondence should be addressed to B.L. (beth.levine@utsouthwestern.edu) or H.W.V. (virgin@wustl.edu). 